Supporting Information

\title{
Effective Heat Dissipation from Color-Converting Plates in High-Power White Light-Emitting-Diodes by Transparent Graphene Wrapping
}

Eden Kim, ${ }^{1, \dagger}$ Hyeon Woo Shim, ${ }^{3, \dagger}$ Sanjith Unithrattil, ${ }^{1, \dagger}$ Yoon Hwa Kim,,${ }^{1, \dagger}$ Hojin Choi, ${ }^{3}$

Ki-Jin Ahn, ${ }^{3}$ Joon Seop Kwak, ${ }^{4}$ Sungmin Kim, ${ }^{2,3}$ Hyeonseok Yoon, ${ }^{2,3,{ }^{*}}$ and Won Bin Im ${ }^{1, *}$

${ }^{1}$ School of Materials Science and Engineering and Optoelectronics Convergence Research Center, Chonnam National University, Gwangju 61186, South Korea.

${ }^{2}$ Alan G. MacDiarmid Energy Research Institute, School of Polymer Science and Engineering, Chonnam National University, Gwangju 61186, South Korea.

${ }^{3}$ Department of Polymer Engineering, Graduate School, Chonnam National University, Gwangju 61186, South Korea.

${ }^{4}$ Department of Printed Electronics Engineering, Sunchon National University, Jeonnam 57922 , South Korea

${ }^{\dagger}$ These authors contributed equally to this work

1*e-mail: imwonbin@jnu.ac.kr

$2^{2}$ e-mail: hyoon@chonnam.ac.kr 


\section{Experimental details}

\section{Materials.}

The starting materials, $\mathrm{B}_{2} \mathrm{O}_{3}$ (Aldrich, $99.99 \%$ ), $\mathrm{Al}_{2} \mathrm{O}_{3}$ (Aldrich, $99.99 \%$ ), $\mathrm{SiO}_{2}$ (Aldrich, $99.99 \%$ ), $\mathrm{K}_{2} \mathrm{CO}_{3}$ (Kojundo, 99\%) and $\mathrm{ZnO}$ (Aldrich, $99.99 \%$ ) were used as obtained without any further purification. The commercial phosphors, SCGS:Eu ${ }^{2+}$ and CASN:Eu ${ }^{2+}$, were purchased from Sony Corporation and Mitsubishi Chemical Corporation, respectively. Copper foil (99.98\%) was purchased from Aldrich. PMMA (MicroChem Corp. 950 PMMA A4, 4\% in anisole) and ferric chloride solution (CE-100, Transene) were used to transfer graphene onto the PGPs.

\section{Preparation of PGPs.}

Glass frits were prepared from the oxides of the constituent cations, viz., $\mathrm{B}_{2} \mathrm{O}_{3}, \mathrm{Al}_{2} \mathrm{O}_{3}, \mathrm{SiO}_{2}$, $\mathrm{K}_{2} \mathrm{O}$, and $\mathrm{ZnO}$ in appropriate proportions to form a $\left(\mathrm{SiO}_{2}-\mathrm{ZnO}-\mathrm{B}_{2} \mathrm{O}_{3}\right)-\mathrm{Al}_{2} \mathrm{O}_{3}-\mathrm{K}_{2} \mathrm{O}$ system, where $\mathrm{SiO}_{2}-\mathrm{ZnO}-\mathrm{B}_{2} \mathrm{O}_{3}$ constitutes $80 \mathrm{wt} \%$ while $\mathrm{Al}_{2} \mathrm{O}_{3}$ and $\mathrm{K}_{2} \mathrm{O}$ constitute 5 and $15 \mathrm{wt} \%$, respectively. The constituents were then mixed and homogenized to form glass frits. The commercial SCGS: $\mathrm{Eu}^{2+}$ was then mixed with $\mathrm{CASN}: \mathrm{Eu}^{2+}$ in an appropriate ratio to obtain the desired color coordinate. The appropriate amount of glass frit was added to the phosphor mixture, and the resulting three-component mixture was homogenized in an agate mortar. The homogenized mixture was then compressed under an axial load of 1.5 tons to form disks, which were then fired at temperatures ranging from 500 to $700^{\circ} \mathrm{C}$. The obtained PGPs were then optically polished and used for further analysis (Opt. Lett. 2013, 38, 3298-3300). 


\section{CVD growth of graphene.}

A home-made CVD system was used to obtain graphene. Graphene was grown on a $25-\mu \mathrm{m}$-thick copper foil in a horizontal quartz tube furnace using methane and hydrogen gases. First, the copper foil was loaded into the quartz tube furnace; then the quartz tube was purged with argon gas and evacuated to $10 \mathrm{mTorr}$. The tube was heated to $1000^{\circ} \mathrm{C}$ with a $40 \mathrm{sccm} \mathrm{H}_{2}$ flow over 30 min, and then a $120 \mathrm{sccm} \mathrm{CH}_{4}$ flow was established in the tube for $30 \mathrm{~min}$ at approximately 2.5 Torr. Lastly, the quartz tube was rapidly cooled to room temperature under $\mathrm{H}_{2}$.

\section{Transfer of graphene onto PGPs.}

To transfer the pristine graphene onto the PGPs, the CVD graphene on the copper substrate was first coated with PMMA solution by spin coating at $6000 \mathrm{rpm}$ for $1 \mathrm{~min}$. The PMMA/graphene thin film was released by etching the underlying copper with ferric chloride solution and rinsed several times in distilled water to completely remove residual impurities. The PMMA/graphene film was then transferred onto a PGP and the PMMA was carefully removed from the G-PGP using aqueous acetic acid solution. The volume ratio of acetic acid to distilled water was 1:1. The resulting graphene was further washed with distilled water and the removal of residual impurities was confirmed by AFM. Two graphene films were used to coat the front and back faces of the PGP. 


\section{Raman spectroscopic analysis of graphene}

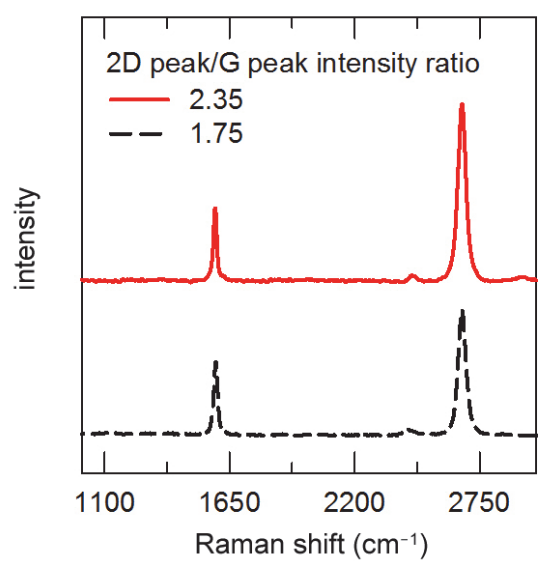

Figure S1. Two representative Raman spectra of the graphene used in this work.

The Raman spectra of the graphene layer have characteristic G and 2D peaks at 1585 and 2675 $\mathrm{cm}^{-1}$, respectively, as shown in Figure S1. Two typical features of single-layer graphene were observed: i) a G-to-2D peak intensity ratio of approximately 0.5 and ii) a symmetric $2 \mathrm{D}$ peak with a full width at half maximum of approximately $32 \mathrm{~cm}^{-1}$. This confirmed that the asproduced CVD graphene consists of a single-layer. Sometimes, a small D peak was observed at $1350 \mathrm{~cm}^{-1}$, which would stem from unwanted defects during the transfer process. 


\section{Thermal conductivity of graphene}

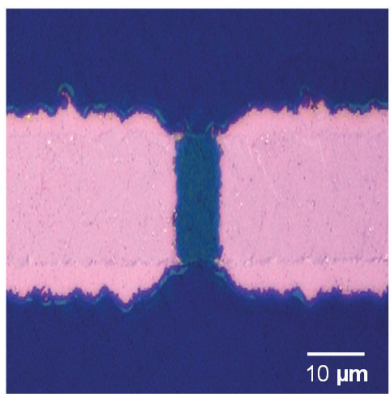

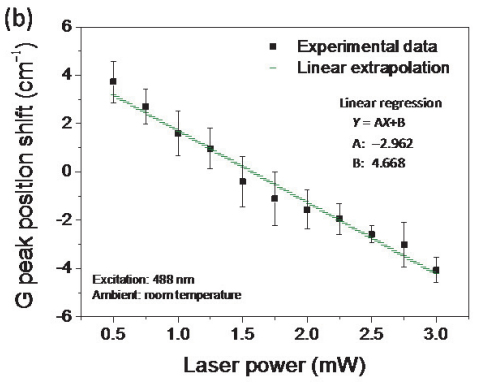

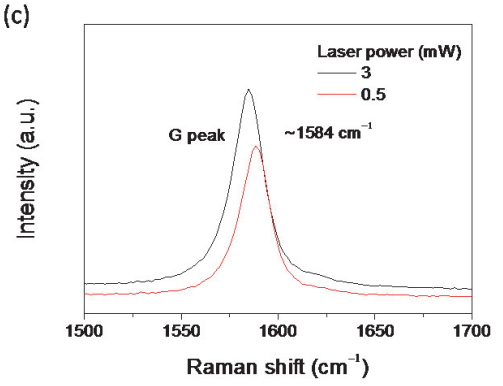

Figure S2. (a) Optical image of CVD graphene $\left(4 \times 20 \mu \mathrm{m}^{2}\right)$ immobilized between two gold microelectrodes on a $\mathrm{Si} / \mathrm{SiO}_{2}$ substrate. (b) Shift in $\mathrm{G}$ peak spectral position vs. change in heating laser power, and (c) G peak region of the Raman spectrum from single-layer graphene recorded at two different excitation laser powers with a $532 \mathrm{~nm}$ excitation; the spectra were recorded at room temperature.

The optical methodology (using Raman spectroscopy) developed by the Balandin group (Nano Lett. 2008, 8, 902-907) was employed, for which the graphene was immobilized between two gold microelectrodes on a silicon wafer with $200 \mathrm{~nm}$ of $\mathrm{SiO}_{2}$ (Figure S2a). The temperature dependence of the frequency of the G peak in the Raman spectra of graphene supported on $\mathrm{Si} / \mathrm{SiO}_{2}$ substrates is plotted in Figure $\mathrm{S} 2 \mathrm{~b}$. The red shift of the Raman $\mathrm{G}$ peak of graphene was a linear function of the incident laser power, as shown in Figure S2b and S2c.

In Figure S2b, the extracted slope $(\mathrm{d} w / \mathrm{d} P)$ was approximately $-2.96 \mathrm{~cm}^{-1} / \mathrm{mW}$. The thermal conductivity $(k)$ was calculated using the formula

$$
k=\chi_{\mathrm{g}}(L / 4 h W)(\mathrm{d} w / \mathrm{d} P)^{-1}
$$

where $\chi_{\mathrm{g}}$ denotes the first-order temperature coefficient of graphene, and $h, W$, and $L$ denote the thickness, width, and length, respectively. The temperature coefficient $\chi_{\mathrm{g}}$ of single-layer 
graphene ranges from $-1.62 \times 10^{-2}$ to $-3.00 \times 10^{-2} \mathrm{~cm}^{-1} / \mathrm{K}$, which defines the temperature dependence of the wavenumber of the Raman G peak. Calizo et al. (Nano Lett. 2007, 7, 26452649) reported a $\chi_{\mathrm{g}}$ value of $-1.62 \times 10^{-2} \mathrm{~cm}^{-1} / \mathrm{K}$ (with $488 \mathrm{~nm}$ excitation on a $\mathrm{SiO}_{2}$ substrate) and Nguyen et al. (Nano Lett. 2011, 5, 5273-5279) reported a $\chi_{\mathrm{g}}$ value of $-3.00 \times 10^{-2} \mathrm{~cm}^{-1} / \mathrm{K}$ (with $633 \mathrm{~nm}$ excitation on a $\mathrm{SiO}_{2}$ substrate). The thermal conductivity of the graphene is calculated to be 978 or $1835 \mathrm{~W} / \mathrm{mK}$ for $-1.62 \times 10^{-2}$ or $-3.00 \times 10^{-2} \mathrm{~cm}^{-1} / \mathrm{K} \chi_{\mathrm{g}}$ value. Cai et al. (Nano Lett. 2010, 10, 1645-1651) examined the thermal properties of suspended and supported single-layer CVD graphene. The calculated thermal conductivities of our graphene appear to be higher than those (500 to $600 \mathrm{~W} / \mathrm{mK}$ ) of the supported graphenes previously reported (Science 2010, 328, 213-216; Nanoscale 2013, 5, 532-536). Of course, the thermal conductivities are lower than those (3000 to $5000 \mathrm{~W} / \mathrm{mK}$ ) of the suspended graphenes (Nano Lett. 2008, 8, 902 907). 


\section{Long-term stability}
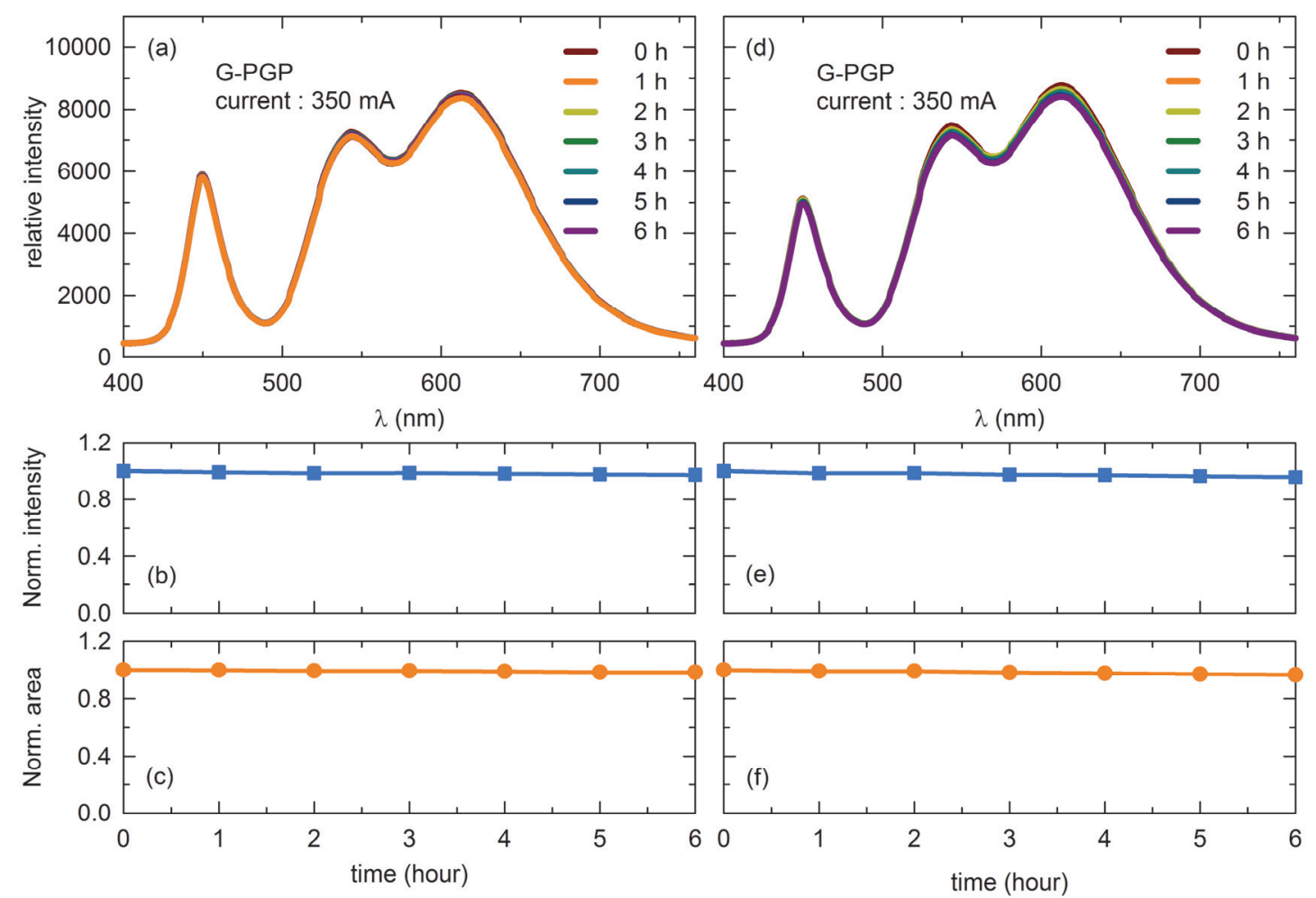

Figure S3. EL spectra of the WLED with G-PGP, measured under an operating current of 350 $\mathrm{mA}$ for $6 \mathrm{~h}$ with a time interval of $1 \mathrm{~h}$ during (a) the first and (d) the second trials, respectively. $(\mathrm{b}, \mathrm{e})$ EL emission intensity and $(\mathrm{c}, \mathrm{f})$ emission area plotted against time. 


\section{Cooling down process}

The heat-diffusion in the PGP was examined by monitoring the cool-down process. Initially, the bottom of the PGP was brought to a temperature of around $180^{\circ} \mathrm{C}$, and was transferred to a frame, maintained at room temperature. The PGP was allowed to cool down under similar conditions to those associated with an LED. The temperature of the upper surface was estimated from the thermal image of the PGP with a time interval of 1 min as shown in Figure S4a and S4b.
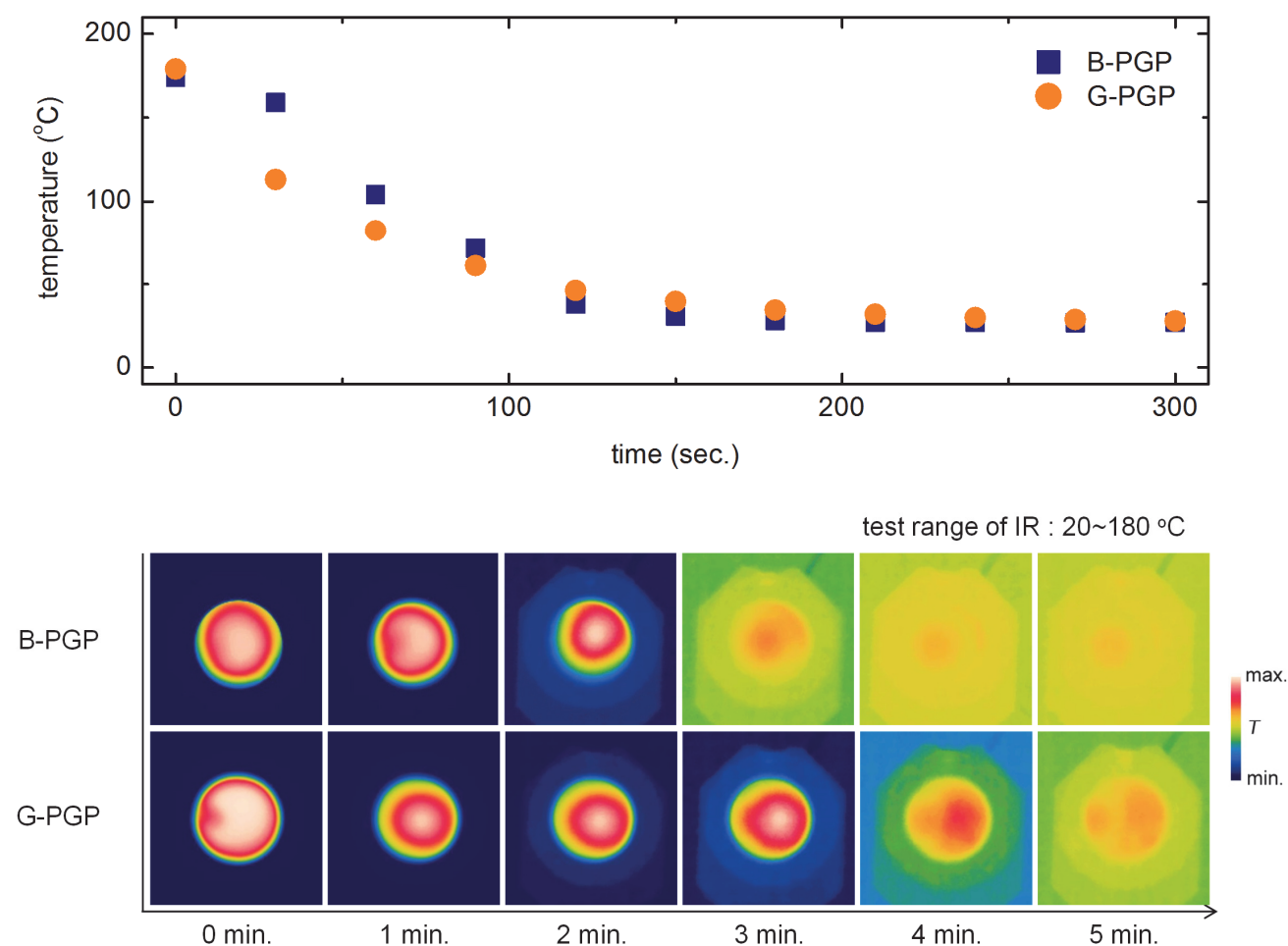

Figure S4. (a) Plot of maximum temperature of the upper surface of B-PGP and G-PGP against time during the cooling process. (b) Thermal imaging of the upper surface of B-PGP and G-PGP during the cooling process. 
The observations can be summarized as follows:

(1) From the beginning of the cooling process to the $120 \mathrm{~s}$ point, the G-PGP showed a much faster cooling rate than the B-PGP. After $120 \mathrm{~s}$, the B-PGP and G-PGP had very similar temperatures, as shown in Figure S4a.

(2) Figure S4b shows the temperature distribution of B-PGP and G-PGP during the cooling process. Both PGPs revealed remarkably different temperature distribution from the $180 \mathrm{~s}(3$ min) duration. Starting from this time, the maximum temperature of both PGPs became almost identical, as plotted in Figure S4a. However, the G-PGP had still hot zones concentrated on the graphene domain, in contrast with the B-PGP. These observations indicate that graphene is still dissipating residual heat effectively on the PGP at the lower temperatures. 


\section{Cellular automata simulation details}

The temperature of each cell is set to zero in the beginning of the analysis. Heat is continuously provided to the G-PGP cells by an excitation source. The temperature change of these cells can be expressed using the following relation,

$$
\Delta T=\frac{Q}{m \times c}
$$

where $Q, m$, and $c$ refer to the heat provided, specific mass, and specific heat, respectively. Then heat flows to the adjacent cells because a temperature gradient develops among the cells. Heat transferred by conduction can be calculated using the following relation,

$$
\frac{d Q}{d t}=\frac{k \times A \times \Delta T(t)}{D}
$$

where $k, A, t$, and $D$ are the thermal conductivity, cross-sectional area, elapsed time, and thickness of the material, respectively. Since the dimensions of all cells were assumed to have the same units, $A$ and $D$ become unity. Time $t$ is also regarded as unity as the analysis method developed in this study is performed iteratively using a non-dimensional discrete time interval.

Heat transferred by convection can be calculated using Eq. 3 .

$$
\frac{d Q}{d t}=H_{C} \times A \times \Delta T(t)
$$

where, $H_{\mathrm{C}}$ denotes the heat transfer coefficient (assumed here to be independent of $T$ ). Each cell obtains thermal energy from the adjacent cells when the temperature of the adjacent cells is higher, and loses energy when its temperature is higher than that of the adjacent cells. Heat transfer by a combination of conduction and convection was considered to build our simulation. Heat conduction occurs within an object or material, or between two objects that are in contact 
with each other; hence, conduction takes place in all components of the analysis domain including air, graphene, and PGP. On the other hand, convection is commonly thought of as the transfer of heat energy by the physical movement of a fluid such as a gas or a liquid. Thus, convection only occurs when at least one of the two adjacent cells is air in the analysis domain. The majority of the heat dissipation from the G-PGP to air (the surrounding) is the result of convection; the effect of radiation is neglected.

Table S1. Representative physical properties of individual components. ${ }^{[a]}$

\begin{tabular}{|c|c|c|c|c|}
\hline component & $\begin{array}{c}\text { specific mass } \\
\left(\mathrm{g} \mathrm{cm}^{-3}\right)\end{array}$ & $\begin{array}{l}\text { specific heat } \\
\left(\mathrm{J} \mathrm{g}^{-1} \mathrm{~K}^{-1}\right)\end{array}$ & $\begin{array}{c}\text { thermal } \\
\text { conductivity } \\
\left(\mathrm{W} \mathrm{m}^{-1} \mathrm{~K}^{-1}\right)\end{array}$ & $\begin{array}{c}\text { heat transfer } \\
\text { coefficient } \\
\left(\mathrm{W} \mathrm{m} \mathrm{m}^{-2} \mathrm{~K}^{-1}\right)\end{array}$ \\
\hline air & $1.2 \times 10^{-3}$ & 1.0 & $2.6 \times 10^{-2}$ & 5.0 \\
\hline graphene & 1.8 & 0.7 & $1.0 \times 10^{3}$ & $2.5 \times 10^{5}$ \\
\hline $\mathrm{PGP}^{[\mathrm{b}]}$ & 2.9 & $2.6 \times 10^{-1}$ & 1.4 & $5.0 \times 10^{2}$ \\
\hline
\end{tabular}

${ }^{[\mathrm{a}]}$ The values were taken from the literature.

${ }^{[b]}$ The phosphor was mixed with glass and thus the mixed ratio was considered to calculate the properties of the PGP. 
Table S2. Adjusted physical parameters of individual components used for the simulation.

\begin{tabular}{|c|c|c|c|c|}
\hline component & $\begin{array}{l}\text { specific mass } \\
\left(\mathrm{g} \mathrm{cm}^{-3}\right)\end{array}$ & $\begin{array}{l}\text { specific heat } \\
\left(\mathrm{J} \mathrm{g}^{-1} \mathrm{~K}^{-1}\right)\end{array}$ & $\begin{array}{c}\text { thermal } \\
\text { conductivity } \\
\left(\mathrm{W} \mathrm{m}^{-1} \mathrm{~K}^{-1}\right)\end{array}$ & $\begin{array}{r}\text { heat transfer } \\
\text { coefficient } \\
\left(\mathrm{W} \mathrm{m}^{-2} \mathrm{~K}^{-1}\right)\end{array}$ \\
\hline air & $1.2 \times 10^{3}$ & 1.0 & $2.6 \times 10^{-2}$ & 5.0 \\
\hline graphene & 1.8 & 0.7 & $1.0 \times 10^{3}$ & $2.5 \times 10^{5}$ \\
\hline PGP & $2.9 \times 10^{6}$ & $2.6 \times 10^{-1}$ & 1.4 & $5.0 \times 10^{2}$ \\
\hline
\end{tabular}




\section{SEM image of PGP with energy dispersive spectra}

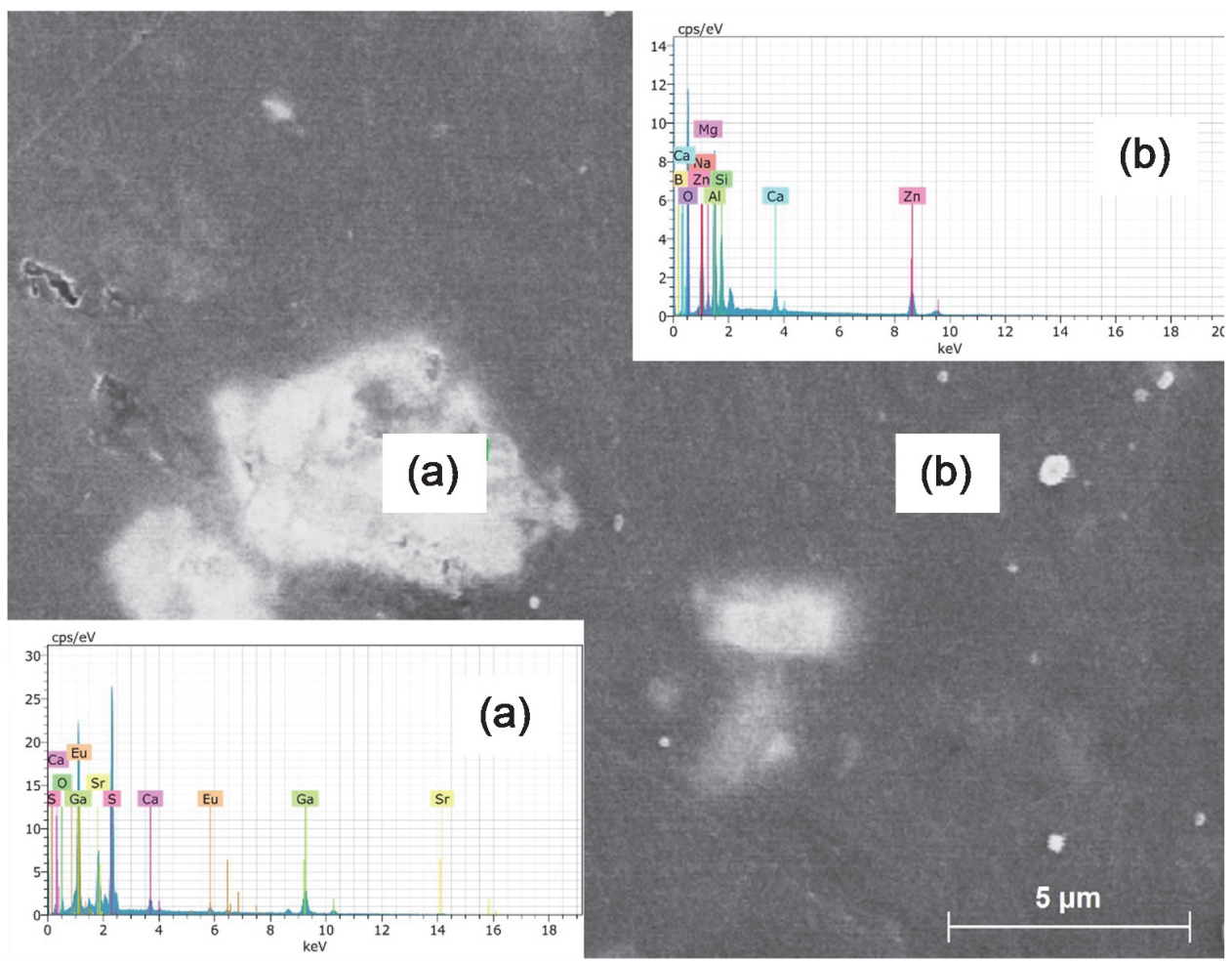

Figure S4. SEM image of PGP. The inset shows the energy dispersive spectra of specific points: (a) the phosphor and (b) glass matrix.

Figure S4 presents an SEM image of the PGP showing the distribution of phosphor in the glass matrix. The insets in Figure S4 display the energy dispersive spectra which indicate the existence of the characteristic phases of phosphor and glass without any decomposition. 


\section{The integrity of the side of G-PGP}

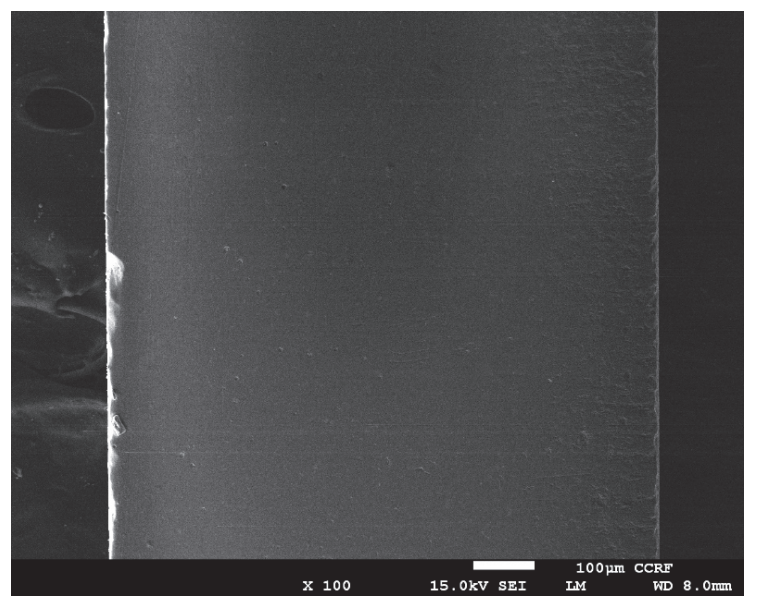

Figure S5. SEM image of the side of the G-PGP.

It is very difficult to observe how the graphene pieces are connected on the side of the PGP. It is believed that the two graphene pieces were well connected via Van der Waals attraction. The restacking of graphene pieces to form graphite is well known. Figure S5 presents the SEM image of the PGP side. There were no significant microstructures, implying that the side of the PGP was well wrapped with graphene without significant defects. 


\section{The EL of G-PGP in comparison with B-PGP}
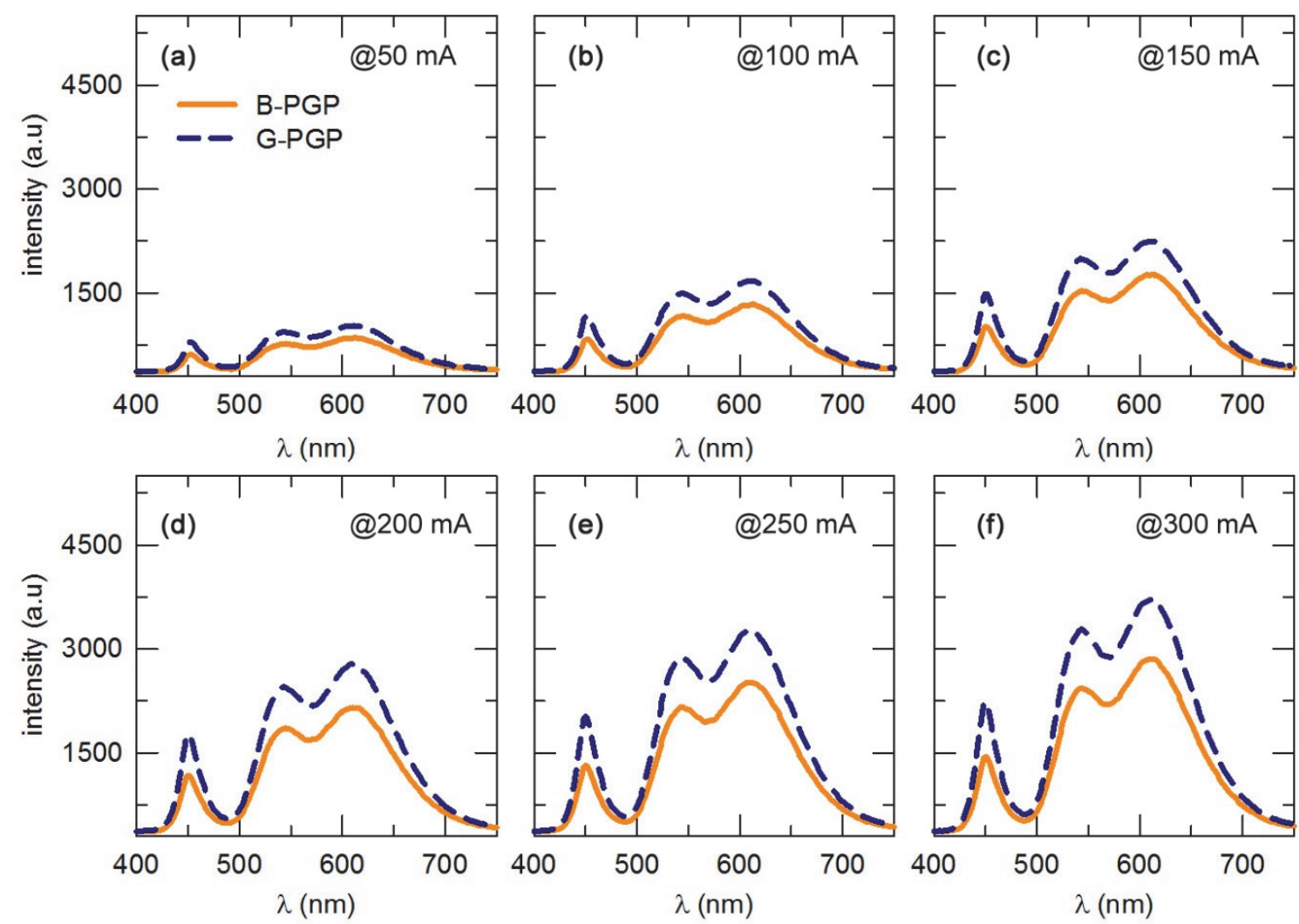

Figure S6. EL spectra of G-PGP in comparison with B-PGP under different forward bias currents:

(a) $50 \mathrm{~mA}$, (b) $100 \mathrm{~mA}$, (c) $150 \mathrm{~mA}$, (d), $200 \mathrm{~mA}$, (e) $250 \mathrm{~mA}$, and (f) $300 \mathrm{~mA}$, respectively. 